Association in 2004. The histopathology examination and TTC staining agree with the AMI diagnosis too. The experiment of rabbits in IR group was in accordance with the recanalisation criteria of IRCA recommended by the Chinese J Cardiol editorial committee in 1991. The injection of Evan's Blue was in coincidence with the recanalisation of IRCA too.

Conclusions AMI-reperfusion rabbit models can be successfully established by the application of this method. It has proved to be very effective.

\section{E0237 EXTRACELLULAR MATRIX ISOLATED FROM MYOCARDIAL TISSUE SUPPORTS THE CARDIOMYOCYTE DIFFERENTIATION OF EMBRYONIC STEM CELLS}

doi:10.1136/hrt.2010.208967.237

Ou Dongbo, Zheng Diangsun, He Yong, Wang Zikuan. Department of Cardiology, Tangdu Hospital Fourth Military Medical University, Xi'an, China

Objective Embryonic stem cells (ESCs) represent an extremely promising cell source for tissue-engineered myocardial tissue. But the role of extracellular matrix in control and guidance of ESCs differentiation and commitment into complex and viable threedimensional (3D) myocardial tissue is still poorly understood. The purpose of this study was to identify and functionally characterise survive and differentiation of ESCs in 3D extracellular matrices isolated from myocardial tissues.

Methods Undifferentiated ESCs were cultured as previously described. Native myocardial tissues isolated from hearts of adult rabbit were treated with trypsin-EDTA and $\mathrm{NaOH}$ to remove cell components to gain acellular matrices. The acellularization of myocardial tissues was documented by haematoxylin-eosin staining (HE staining). Then ESCs were seeded onto the acellular matrices. Cell density and distribution were assessed by HE staining, cardiac transcription factors such as GATA4, NKX2.5, and atrial natriuretic factor (ANF) were evaluated by RT-PCR, and cardiac markers such as $\boldsymbol{\alpha}$-actinin, troponin I, and connexin 43 were evaluated by immunocytochemistry. Ultrastructural analysis was examined under a transmission electron microscope.

Results The acellular matrices were incompletely covered by cells at 1 week, and the interstitiums of matrices were densely repopulated by cells at 2 weeks post seeding, indicating the rapid growth and expansion of these cells in the extracellular matrices and the formation of live constructs. Cardiac transcription factors GATA4 and NKX2.5 began to express on day10, but the expression of ANF was detected on day 12 . The constructs were positively immunostained with $\alpha$-actinin, troponin I, and connexin 43 at 2 weeks post seeding. Ultrastructural analysis indicates that cells in constructs exhibited the morphological features including sarcomeres, abundant glycogen and mitochondria and nascent junctional complexes. Conclusions These results indicate that extracellular matrices have an important role to promote the differentiation of embryonic stem cells into cardiomyocytes. Such cell differentiation with extracellular matrices may be useful in forming tissue-engineered myocardial tissue to repair specific damaged hearts.

\section{e0238 EFFECTS OF COMBINED ATORVASTATIN/COENZYME 010 ON MYOCARDIAL FIBROSIS AND MALONDIALDEHYDE (MDA) AND SUPEROXIDE DISMUTASE (SOD) IN RAT OF HEART FAILURE}

doi:10.1136/hrt.2010.208967.238

${ }^{1}$ Zhao Dongming, ${ }^{2}$ Yang Ping. ${ }^{1}$ Affiliated Hospital of Beihua University; ${ }^{2}$ China-japan Union Hospital of Jilin University

Objective To observe the effects of Combined Atorvastatin/Coenzyme Q10 on myocardial fibrosis and MDA and SOD in Rat of heart failure, to explore the protective effect of Combined Atorvastatin/ Coenzyme Q10 on the heart of the rat.

Methods Female Wister rats were subjected to the ligation of the anterior descending branch of the left coronary artery or sham operation during halothane anaesthesia. The rat heart failure models were established after 6 weeks. These rats were divided into four groups ( $n=6$ each group): Sham operated group; model group; model group-atorvastatin, Rats in this treatment group were administrated by gastric perfusion of atorvastatin $(10 \mathrm{mg} / \mathrm{kg} /$ day); model group-atorvastatin-Coenzyme Q10. Rats in this treatment group were administrated by gastric perfusion of atorvastatin $(10 \mathrm{mg} / \mathrm{kg} /$ day) and Coenzyme Q10 (30 mg/kg/day). All treatment regimens were initiated 6 weeks after surgery and continued for 5 weeks. After Masson stain, myocardial fibrosis of these rats were observed by light microscope. The activity of serum SOD and the content of serum MDA were detected by biochemical methods.

Results Compared with that in model group, myocardial fibrosis level and MDA content was decreased in model group-atorvastatin $(p<0.01)$. At the same time, the activity of SOD was increased $(p<0.01)$. Compared with that in model group-atorvastatin, myocardial fibrosis level and MDA content was descreased in model group-atorvastatin-Coenzyme $\mathrm{Q} 10(\mathrm{p}<0.05)$. At the same time, the activity of SOD was increased $(\mathrm{p}<0.05)$.

Conclusion Compared with atorvastatin, Combined atorvastatin/ coenzyme Q10 could protect the myocardium further, and MAD and SOD maybe were involved in this process.

\section{e0239 N-TERMINAL PRO-BRAIN NATRIURETIC PEPTIDE (NT- PROBNP): A POTENTIAL DIAGNOSTIC BIOMARKER FOR PREDICTING CARDIAC DYSFUNCTION IN PATIENTS WITH LIVER DISEASES}

doi:10.1136/hrt.2010.208967.239

${ }^{1}$ Wang Ling, ${ }^{1}$ Geng Jiabao, ${ }^{1}$ Chang Yibin, 'Li Tong, ${ }^{2}$ Matsumori Akira, 'Zhuang Hui. ${ }^{1}$ Peking University Health Science Center; ${ }^{2}$ Department of Cardiovascular Medicine Kyoto University Japan

Background NT-proBNP has emerged as a powerful diagnostic and prognostic biomarker in heart disease. Studies showed that NTproBNP is a sensitive biomarker for identifying patients with heart failure caused by hepatitis $\mathrm{C}$ virus (HCV) related myocarditis. The aim of this study was to investigate the relationship between the elevated concentration of NT-proBNP and liver diseases.

Methods 223 serum samples from blood donors were collected as a control group, and 644 samples were obtained from patients infected by hepatitis viruses including 493 HBV (364 chronic hepatitis B, 86 hepatocellular carcinoma and 43 liver cirrhosis) and $151 \mathrm{HCV}$. All samples were assayed with an Elecsys immunoassay analyser for NT-proBNP concentration.

Results The mean concentration of NT-proBNP in the control group was $21.77 \pm 15.56 \mathrm{pg} / \mathrm{ml}$ which showed no significant variation with age or gender. Both the mean value and the rate of abnormality of NT-proBNP were significantly higher for the HBV and HCV infected groups as compared to the control group. The mean NT-proBNP value $(380.24 \mathrm{pg} / \mathrm{ml})$ and abnormality rate $(38.41 \%)$ in the HCV group were higher than that of HBV group. For samples from patients with HBV related hepatic disease/pathology, the mean NTproBNP value $(517.19 \mathrm{pg} / \mathrm{ml})$ and abnormality rate $(58.14 \%)$ were the highest in the liver cirrhosis group.

Conclusions The elevation of NT-proBNP in patients with progressed liver disease in this study suggested the presence of cardiac dysfunction. 